\title{
Hétéro-nucléation de cristaux de neige carbonique au débouchage d'une bouteille de champagne
}

Gérard Liger-Belair (gerard.liger-belair@univ-reims.fr) et Daniel Cordier

Équipe Effervescence, Champagne et Applications

Groupe de Spectrométrie Moléculaire et Atmosphérique (UMR CNRS 7331),

Université de Reims Champagne-Ardenne, 51687 Reims

Le débouchage d'une bouteille

de champagne permet de revisiter

la physique des changements

de phase qui accompagnent

la détente adiabatique du volume

de gaz sous pression dans le col

de la bouteille.

On s'intéresse ici aux phénomènes

de condensation qui apparaissent

dans le sillage du bouchon

qui saute, en fonction de la

température de ladite bouteille.

Pour les bouteilles stockées

à $20^{\circ} \mathrm{C}$, un panache bleu azur

apparait dans le col de la bouteille

et dans le sillage du bouchon

qui saute. Il s'évanouit au bout

de quelques millisecondes.

On montre que ce panache bleu

est la signature de la transformation

en cristaux de neige carbonique

et par nucléation hétérogène,

de la vapeur de dioxyde

de carbone présente dans le col

de la bouteille.
En moyenne, ce sont près de dix bouchons de champagne qui sautent chaque seconde à l'échelle du globe ! Et ce chiffre explose le jour de la Saint-Sylvestre. Le phénomène de condensation qui accompagne le débouchage d'une bouteille de champagne froide a déjà été décrit dans la littérature scientifique [1-3]. Nous avons néanmoins souhaité le revisiter à l'aide d'une caméra rapide, afin d'examiner en détail le rôle de la température du champagne. Pour ce faire, nous disposions de trente bouteilles de champagne rosé. 48 heures avant de réaliser nos observations, trois lots de dix bouteilles furent stockés respectivement à 6, 12 et $20^{\circ} \mathrm{C}$.

La figure 1 illustre les phénomènes de condensation qui surviennent au voisinage $\mathrm{du}$ bouchon qui saute, en fonction de la température de la bouteille. Au moment du débouchage, la détente du volume de dioxyde de carbone gazeux, initialement sous pression dans le col de la bouteille, s'accompagne d'une chute de sa température. Le nuage de gaz qui se détend refroidit l'air ambiant et la vapeur d'eau qu'il contient. En se refroidissant, la vapeur d'eau se condense sous la forme d'un brouillard de minuscules gouttelettes. C'est la diffusion de la lumière ambiante par ces gouttelettes - de façon quasi isotrope dans l'espace et pour toutes les longueurs d'onde du spectre visible - qui confere au panache de condensation une teinte blanchâtre. Ce mode de diffusion de la lumière, lorsque la taille des objets qui diffusent est comparable ou supérieure aux longueurs d'onde de la lumière ambiante, correspond au régime de Mie. Cette description est bien conforme à ce que l'on peut observer lors du débouchage des bouteilles stockées à 6 et à $12^{\circ} \mathrm{C}$ (figs 1 a et $1 \mathrm{~b}$ ). Cependant, dans le cas de la bouteille stockée à température ambiante, pourquoi le nuage de condensation apparait-il directement dans le goulot de la bouteille et pourquoi est-il bleu azur et non pas blanc (fig. 1c) ? La clé de l'énigme se trouve dans la relation qui existe entre la température des gaz qui se détendent au moment du débouchage et la température initiale de la bouteille.

\section{La pression dans une bouteille augmente avec sa température}

Suite à une seconde fermentation alcoolique en bouteille close (la prise de mousse), les vins de Champagne se chargent en dioxyde de carbone $\left(\mathrm{CO}_{2}\right)$. En effet, tout comme l'éthanol, le $\mathrm{CO}_{2}$ est un sousproduit de la transformation des sucres par les levures. Conformément à la loi de Henry, la concentration $c_{L}$ de $\mathrm{CO}_{2}$ dissous dans le vin est proportionnelle à la pression partielle $P_{B}$ de $\mathrm{CO}_{2}$ dans le col de bouteille : $c_{L}=k_{H} P_{B}(1)$, où $k_{H}$ est la constante de Henry du $\mathrm{CO}_{2}$ dans le vin.

Dans une solution aqueuse composée à près de $87 \%$ d'eau en volume, comme le champagne, le $\mathrm{CO}_{2}$ est très soluble. La constante de Henry du $\mathrm{CO}_{2}$ y est d'environ $1,5 \mathrm{~g} / \mathrm{l} / \mathrm{bar}$ à $20^{\circ} \mathrm{C}$, alors qu'elle n'est que de l'ordre de $20 \mathrm{mg} / \mathrm{l} / \mathrm{bar}$ pour l'oxygène, par exemple. Or, la dépendance exponentielle en température de la constante de Henry d'un gaz en solution aqueuse induit une très forte sensibilité de sa solubilité avec la température (plus la 

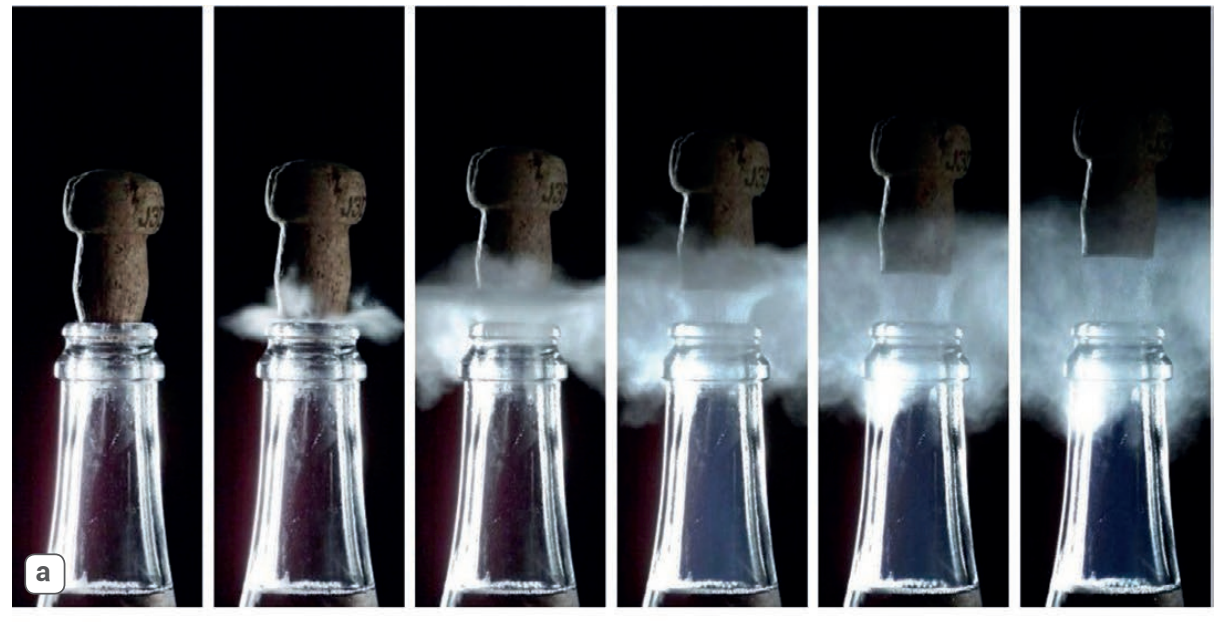

solution est froide et plus le gaz y est soluble). La pression partielle du gaz carbonique dans la bouteille sera donc en retour inévitablement très sensible à la température de ladite bouteille.

Par application de la loi de Henry et de celle des gaz parfaits, on peut calculer la pression de gaz carbonique qui règne dans une bouteille en fonction de sa température $T$. En tenant compte de l'étape qui consiste à retirer le dépôt de levures mortes de la bouteille sous l'effet de la pression après la prise de mousse, puis à reboucher immédiatement ladite bouteille avec un bouchon en liège, la pression de gaz carbonique qui règne dans une bouteille de champagne prête à être dégustée s'exprime comme suit [4] :

$P_{B} \approx n_{T} k_{H}(R T)^{2} V_{L} /\left(V_{G}+k_{H} R T V_{L}\right)^{2}(2)$, où $n_{T}$ est le nombre de moles de $\mathrm{CO}_{2}$ piégées dans la bouteille en fin de fermentation, $V_{L}$ est le volume de champagne, $V_{G}$ est le volume du ciel gazeux dans le col de bouteille et $R$ est la constante des gaz parfaits.

Il existe, bien entendu, d'autres espèces gazeuses que le $\mathrm{CO}_{2}$ en équilibre thermodynamique dans une bouteille de champagne (comme le diazote, l'eau ou l'éthanol). Cependant, compte tenu de la très grande quantité de $\mathrm{CO}_{2}$ produite lors de la seconde fermentation ( 9 grammes par bouteille, soit l'équivalent de cinq litres de gaz carbonique sous une pression de un bar et à $20^{\circ} \mathrm{C}$ ), la pression partielle des autres espèces gazeuses en présence est négligeable comparée à celle du $\mathrm{CO}_{2}$. Nous reviendrons néanmoins sur le cas particulier de l'eau, qui va s'avérer jouer un rôle majeur lors du débouchage.
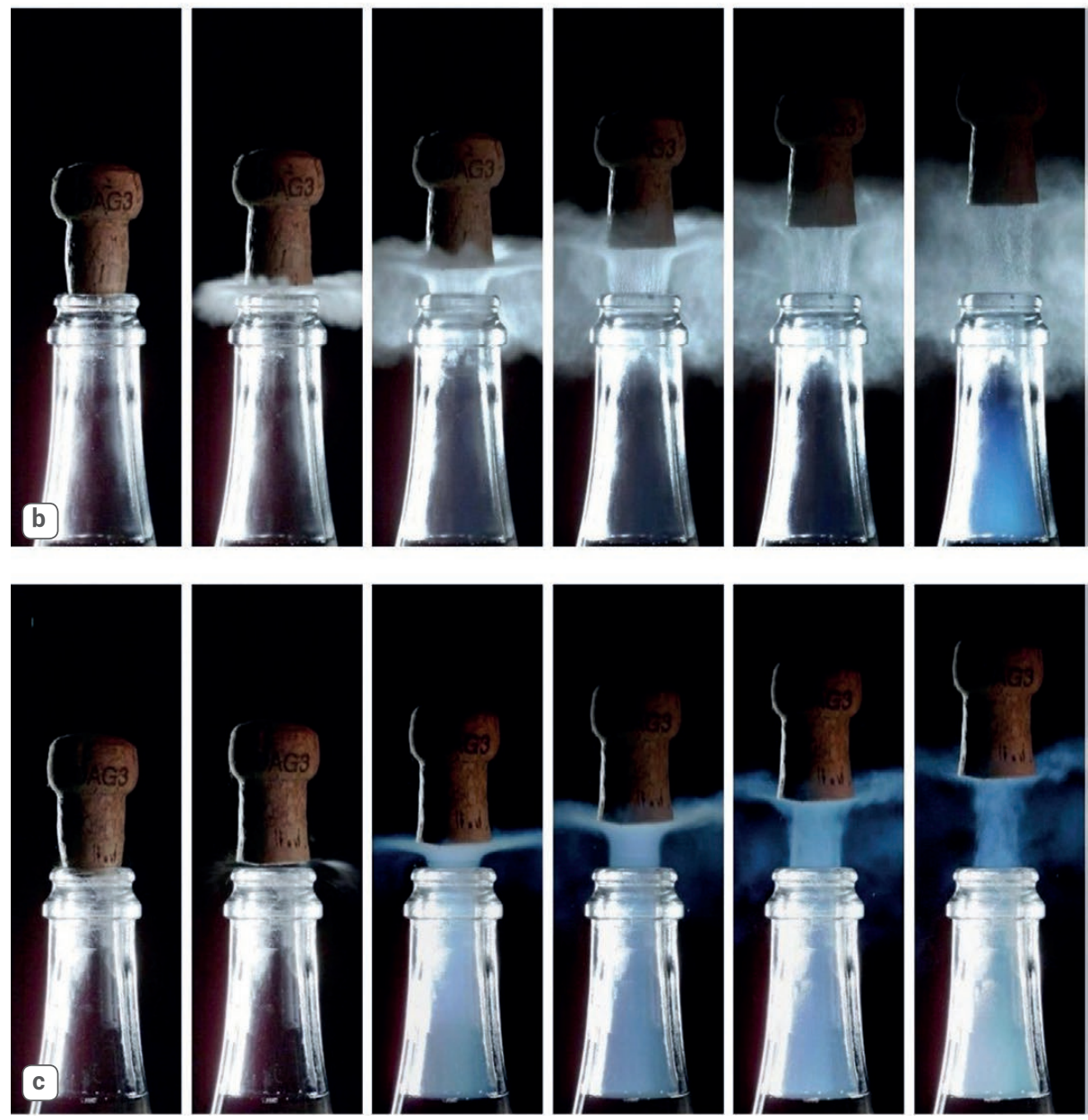

1. Observation, à l'aide d'une caméra rapide (Phantom Flex - Vision Research, USA), du nuage de condensation qui accompagne le débouchage de trois bouteilles stockées respectivement à $6^{\circ} \mathrm{C}(\mathrm{a}), 12^{\circ} \mathrm{C}(\mathrm{b})$, et $20^{\circ} \mathrm{C}$ (c). L'intervalle de temps entre deux images successives est de $400 \mu \mathrm{s}$.

\section{Gros plan sur la détente adiabatique}

Lorsque le bouchon saute, le volume de gaz sous pression dans le col de la bouteille se détend suffisamment brutalement pour qu'il n'y ait pas d'échange de chaleur. Sa température chute alors de façon concomitante pour atteindre une température $T_{f}$ dictée par la relation ci-dessous

$$
T_{f}=T \times\left(\frac{P_{0}}{P_{B}}\right)^{\frac{\gamma-1}{\gamma}}
$$

où $T$ est la température initiale qui règne 
dans la bouteille, $P_{0}$ est la pression ambiante (de l'ordre de $10^{5} \mathrm{~Pa}$, soit 1 bar), $P_{B}$ est la pression du gaz qui règne dans la bouteille bouchée, et $\gamma$ est l'indice adiabatique du gaz défini comme le rapport de ses capacités calorifiques à pression et à volume constants (dans le cas présent il s'agit bien entendu du $\mathrm{CO}_{2}$, dont l'indice $\gamma$ vaut environ 1,3$)$.

Sur le graphique de la figure 2, on peut voir, en fonction de la température initiale $T$ d'une bouteille de champagne de $75 \mathrm{cl}$, la pression $P_{B}$ de gaz carbonique qui règne en son sein lorsqu'elle est encore bouchée, ainsi que la température théorique $T_{f}$ atteinte par le volume de gaz initialement sous pression dans le col de la bouteille après la détente adiabatique (lorsqu'il est revenu à la pression ambiante de 1 bar). De façon assez contre-intuitive, une fois la détente adiabatique réalisée, la température du gaz détendu chute à une valeur d'autant plus basse que la température initiale de la bouteille est élevée. En théorie, le volume de gaz qui se détend hors d'une bouteille stockée à $20^{\circ} \mathrm{C}$ voit sa température chuter à presque $-90{ }^{\circ} \mathrm{C}$ ! Que deviennent donc les différentes espèces gazeuses en présence dans un environnement aussi glacial ?

\section{Des cristaux de neige carbonique}

Le diagramme de phase pression-température du $\mathrm{CO}_{2}$ apparait sur la figure 3 . Sous une pression de $1 \mathrm{bar}$, on constate que le $\mathrm{CO}_{2}$ est un gaz au-dessus d'une température de $-78,5{ }^{\circ} \mathrm{C}$. En dessous de cette température et sous la pression ambiante de 1 bar, le $\mathrm{CO}_{2}$ existe sous sa forme solide (la neige carbonique). Le tableau 1 présente une synthèse des paramètres du mélange gazeux binaire $\mathrm{CO}_{2} / \mathrm{H}_{2} \mathrm{O}$ après le débouchage, une fois la détente adiabatique

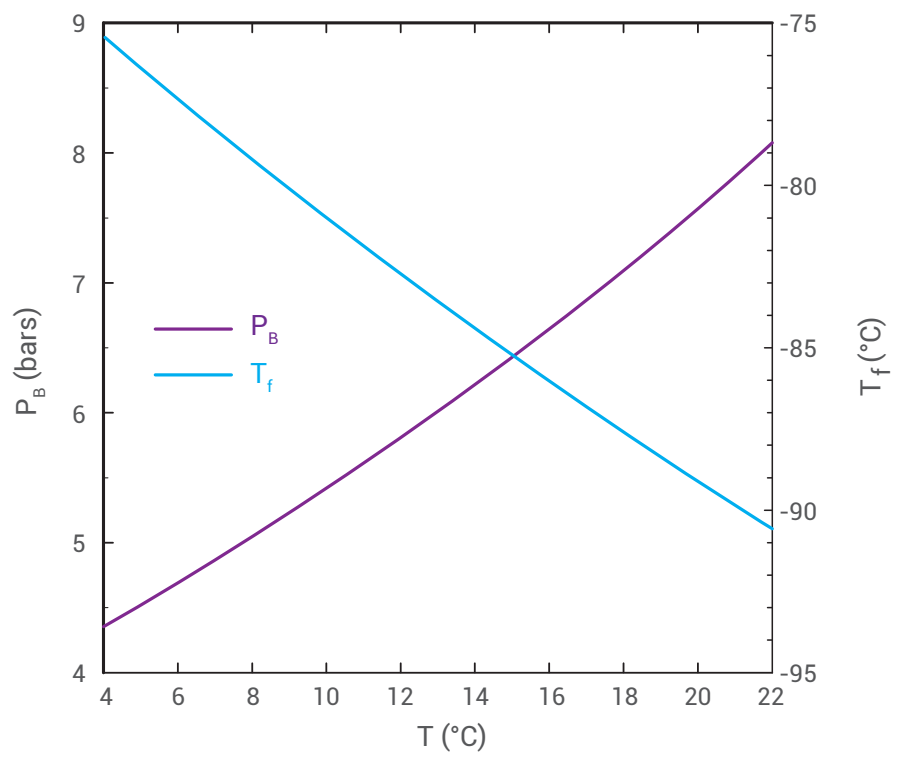

2. Pression partielle de $\mathrm{CO}_{2}$ qui règne dans la bouteille encore bouchée (en violet) et température atteinte par le nuage de gaz carbonique qui se détend lorsque le bouchon saute (en bleu), en fonction de la température initiale de la bouteille.

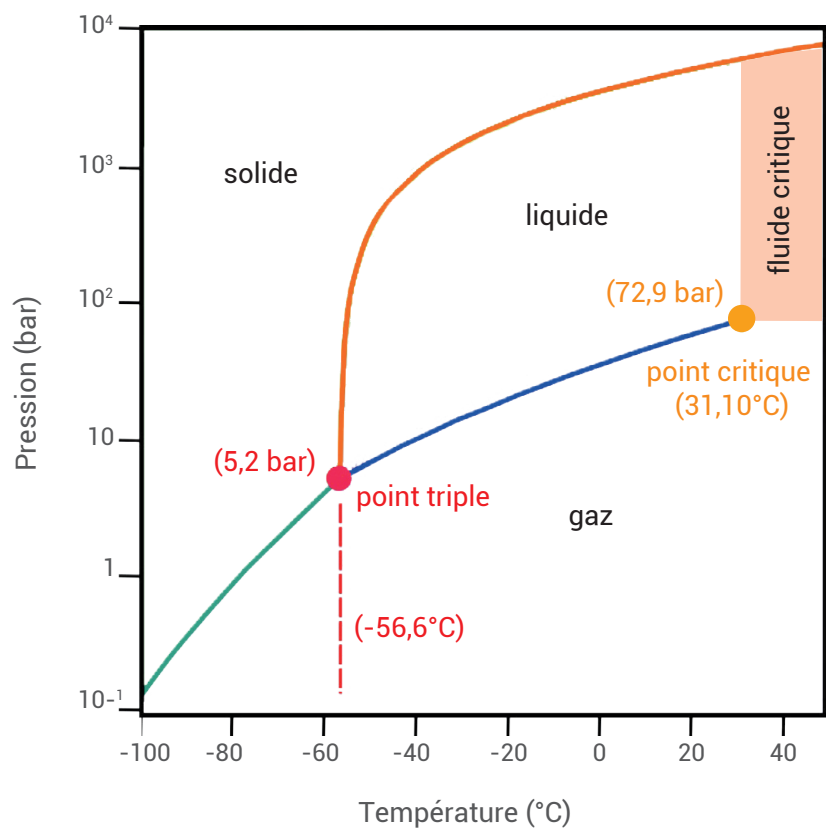

3. Diagramme de phase pression-température du dioxyde de carbone $\left(\mathrm{CO}_{2}\right)$. 

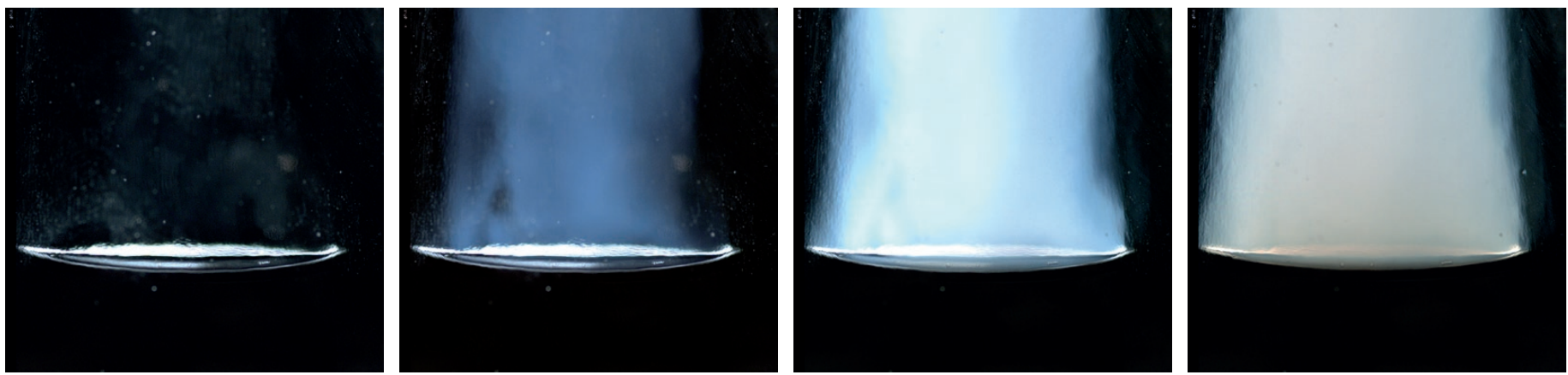

4. Après la détente adiabatique, dans le goulot de la bouteille stockée à $20^{\circ} \mathrm{C}$, on constate que le nuage de condensation vire progressivement du bleu au blanc-gris. 83 s séparent chaque cliché.

réalisée et en fonction de la température initiale de la bouteille. Pour les bouteilles initialement à $6^{\circ} \mathrm{C}$, la température du mélange gazeux qui s'en échappe chute à environ $-77^{\circ} \mathrm{C}$. Il ne fait donc pas encore assez froid pour condenser les vapeurs de $\mathrm{CO}_{2}$ en neige carbonique. Pour les bouteilles stockées à 12 et à $20^{\circ} \mathrm{C}$, la température des gaz qui se détendent chute respectivement à grosso modo -82 et $-90^{\circ} \mathrm{C}$, et donc sous la valeur seuil de $-78,5^{\circ} \mathrm{C}$. Cependant, compte tenu de leur taux de nucléation homogène quasiment nul, les cristaux de neige carbonique ne peuvent tout simplement pas se former. C'est à cette étape qu'un autre acteur du système entre en jeu : la vapeur d'eau, présente dans le col de la bouteille. En effet, le ciel gazeux de la bouteille encore bouchée contient de la vapeur d'eau en équilibre avec le champagne qui, rappelons-le, est formé à près de $87 \%$ d'eau en volume. Lorsque le bouchon saute, la température du mélange gazeux binaire $\mathrm{CO}_{2} / \mathrm{H}_{2} \mathrm{O}$ chute à plusieurs dizaines de degrés sous la température de congélation de l'eau. Il fait si froid que la vapeur d'eau se transforme en cristaux de glace d'eau par nucléation homogène (sans l'aide d'un support solide, compte tenu des énormes coefficients de sursaturation de la vapeur d'eau). Ce sont donc les cristaux de glace d'eau, apparaissant par nucléation homogène dans le col de la bouteille, qui servent de noyaux solides pour la nucléation hétérogène du gaz carbonique en neige carbonique [4]. Des phénomènes similaires ont été mis en évidence dans le mélange gazeux qui se détend dans les tuyères des moteurs de fusées [5]. Là aussi, les gaz les plus facilement condensables (en général l'eau et le $\mathrm{CO}_{2}$ ) servent de noyaux solides qui permettent la condensation des espèces telles que le dioxygène et le diazote, présentant des températures de condensation bien plus basses.

\section{De la diffusion de Rayleigh à celle de Mie}

Le panache bleu azur qui apparait dans le sillage du bouchon qui saute et dans le col de la bouteille serait donc la signature de la transformation des vapeurs de $\mathrm{CO}_{2}$ en cristaux de neige carbonique. Mais pourquoi ce panache est-il bleu, alors que le nuage de condensation de la vapeur d'eau ambiante qui caractérise le débouchage des bouteilles plus froides apparait plutôt blanc ? Selon la théorie de la diffusion, si la taille des cristaux qui diffusent la lumière demeure très inférieure aux longueurs d'onde du spectre de la lumière ambiante, la diffusion sera plus efficace pour les petites longueurs d'onde du spectre, et donc pour la lumière bleue. Ce mode de diffusion de la lumière par des particules plus petites que les longueurs d'onde incidentes est connu sous le nom de diffusion de Rayleigh. C'est le même phénomène qui explique d'ailleurs pourquoi le ciel nous apparait bleu, les molécules qui composent l'atmosphère de notre planète étant bien plus petites que les longueurs d'onde de la lumière visible. Si le nuage de condensation du gaz carbonique ne blanchit pas hors du goulot de la bouteille, c'est parce que les cristaux de neige carbonique qui le constituent ne peuvent pas croitre suffisamment pour atteindre des tailles micrométriques qui le feraient paraitre blanc. En effet, l'air ambiant agit comme un immense réservoir thermique. Très vite la température du nuage de condensation remonte au-dessus de $-78,5^{\circ} \mathrm{C}$, et les cristaux de neige carbonique se subliment pour se transformer en gaz carbonique. Au bout de quelques millisecondes le nuage bleu s'évanouit audessus du goulot, comme sur la figure 1 . Cependant, confiné dans le goulot de la bouteille, le nuage de condensation est protégé du réservoir thermique un peu plus longtemps. Les cristaux de neige carbonique sont donc susceptibles de croitre autour de leur cœur de glace d'eau. Ils atteignent alors des tailles micrométriques et le nuage de condensation change progressivement de couleur. Il vire du bleu profond au blanc-gris, comme on peut clairement le constater sur la figure 4.

\section{Conclusion}

Le débouchage d'une bouteille de champagne, comble de la frivolité pour certains, permet de revisiter la physique des changements de phase et des jets supersoniques sous-détendus. Les phénomènes de condensation qui surviennent dans le sillage d'un bouchon de champagne qui saute, ainsi que le réseau d'ondes de choc qui l'accompagne, se révèlent être assez proches de situations mises en évidence dans l'industrie aéronautique et notamment dans les tuyères des réacteurs d'avions ou de fusées. Nous poursuivons nos investigations en examinant les détails du tout premier millième de seconde qui suit le débouchage, à l'aide de techniques telles que la strioscopie par exemple.

\section{Références}

1・ R. Batt, "Pop! Goes the champagne bottle cork", Journal of Chemical Education, 48 (1971) 71.

2• M. Volmer et K.-P. Möllmann, Physics Education, 45 (2012) 608-615.

3. G. Liger-Belair et al., Journal of Food Engineering, 116 (2013) 78-85.

4- G. Liger-Belair et al., Scientific Reports, 7 (2017) 10938

5• B.E. Wyslouzil et al., Journal of Chemical Physics, 113 (2000) 7317-7329. 